\title{
A Radiation-Triggered Surveillance System for UF6 Cylinder Monitoring
}

\section{September 2016}

Michael Curtis and Mitchell Myjak

Pacific Northwest National Laboratory

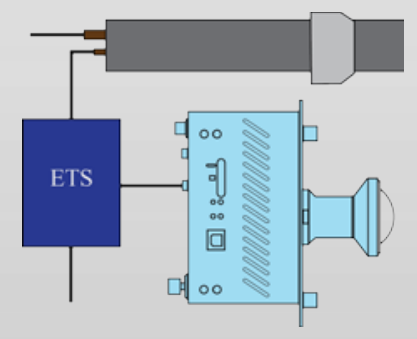




\title{
DISCLAIMER
}

This report was prepared as an account of work sponsored by an agency of the United States Government. Neither the United States Government nor any agency thereof, nor Battelle Memorial Institute, nor any of their employees, makes any warranty, express or implied, or assumes any legal liability or responsibility for the accuracy, completeness, or usefulness of any information, apparatus, product, or process disclosed, or represents that its use would not infringe privately owned rights. Reference herein to any specific commercial product, process, or service by trade name, trademark, manufacturer, or otherwise does not necessarily constitute or imply its endorsement, recommendation, or favoring by the United States Government or any agency thereof, or Battelle Memorial Institute. The views and opinions of authors expressed herein do not necessarily state or reflect those of the United States Government or any agency thereof.

\author{
PACIFIC NORTHWEST NATIONAL LABORATORY \\ operated by \\ BATTELLE \\ for the \\ UNITED STATES DEPARTMENT OF ENERGY \\ under Contract DE-AC05-76RL01830 \\ Printed in the United States of America \\ Available to DOE and DOE contractors from the \\ Office of Scientific and Technical Information, \\ P.O. Box 62, Oak Ridge, TN 37831-0062; \\ ph: (865) 576-8401 \\ fax: (865) 576-5728 \\ email: reports@adonis.osti.gov \\ Available to the public from the National Technical Information Service \\ 5301 Shawnee Rd., Alexandria, VA 22312 \\ ph: (800) 553-NTIS (6847) \\ email: orders@ntis.gov <http://www.ntis.gov/about/form.aspx> \\ Online ordering: http://www.ntis.gov
}




\section{A Radiation-Triggered Surveillance System for UF6 Cylinder Monitoring}

Michael Curtis and Mitchell Myjak

Pacific Northwest National Laboratory

September 2016

Prepared for

U.S. Department of Energy

under Contract DE-AC05-76RL01830

Pacific Northwest National Laboratory

Richland, Washington 99352 



\section{Acknowledgments}

Funding for the preparation of this document was provided by the U.S. Department of Energy National Nuclear Security Administration Office of Nonproliferation and International Security in support of the National Nuclear Security Administration Next Generation Safeguards Initiative. Pacific Northwest National Laboratory wishes to thank the Next Generation Safeguards Initiative for their input, support, and guidance in preparing this document.

The authors wish to thank Benjamin McDonald of Pacific Northwest National Laboratory for developing the GADRAS models used in the gamma count rate simulations. 



\section{Executive Summary}

This report provides background information and representative scenarios for testing a prototype radiation-triggered surveillance system at an operating facility that handles uranium hexafluoride $\left(\mathrm{UF}_{6}\right)$ cylinders. The safeguards objective is to trigger cameras using radiation, or radiation and motion, rather than motion alone, to reduce significantly the number of image files generated by a motion-triggered system.

The authors recommend the use of radiation-triggered surveillance at all facilities where cylinder paths are heavily traversed by personnel. The International Atomic Energy Agency (IAEA) has begun using surveillance cameras in the feed and withdrawal areas of gas centrifuge enrichment plants (GCEPs). ${ }^{1}$ The cameras generate imagery using elapsed time or motion, but this creates problems in areas occupied 24/7 by personnel. Either motion-or-interval-based triggering generates thousands of review files over the course of a month. Since inspectors must review the files to verify operator material-flow-declarations, a plethora of files significantly extends the review process.

Undeclared production, an instance where operators use undeclared, natural $\mathrm{UF}_{6}$ to produce undeclared product in the form of low-enriched $\mathrm{UF}_{6}$, remains a safeguards quandary. This is primarily because process cylinders are under neither seal nor surveillance at most facilities. The IAEA's New Model Safeguards Approach recommends the sealing of all empty and full cylinders or the use of surveillance in the process area as a means of detecting unreported production. ${ }^{2}$ Placing all cylinders and process stations under seal at a commercial facility is very time consuming and would require a continual inspector presence so as not to interfere with operations. There is no easy means of sealing all cylinders at their stations, but the authors believe inspectors can apply surveillance in areas of high activity if the cameras use radiation rather than motion for triggering.

Surveillance is a better alternative than seals for monitoring feed and withdrawal stations. Cameras have the potential to record the passage of all cylinders - declared and undeclared. It provides inspectors with an accurate, independent, long-term, flow balance to compare with operator cylinder-flow declarations.

The IAEA's Next Generation Surveillance System (NGSS) is rapidly replacing early digital surveillance systems the IAEA has employed since the mid-1990s. NGSS and its concomitant review software provide an extremely flexible surveillance platform the IAEA has not fully exploited. The authors' simulation data indicate it should not be difficult to use radiation to record cylinder movements and possibly even facilitate cylinder identification. The use of at least two cameras provides $100 \%$ redundancy in the event one camera fails, camera view is blocked, or camera jarring changes the field of view. Two or more cameras also facilitate resolving discrepancies between operator declarations and inspector verifications.

The primary advantage of radiation-triggered surveillance is the opportunity to obtain full-time cylinder throughput verification versus what presently amounts to part-time verification. Cost savings should be substantial, as the IAEA presently uses frequent unannounced inspections to verify cylinder-throughput declarations. The use of radiation-triggered surveillance allows the IAEA to implement less frequent unannounced inspections for the purpose of flow verification, but its principal advantage is significantly shorter and more effective inspector video reviews. 


\section{Table of Contents}

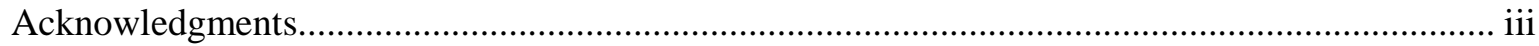

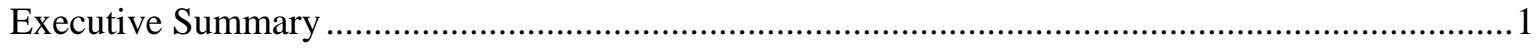

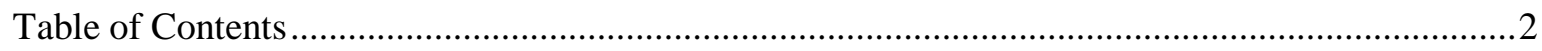

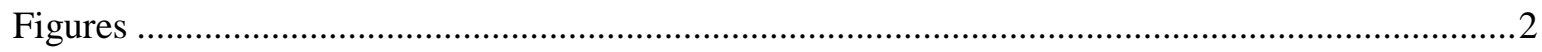

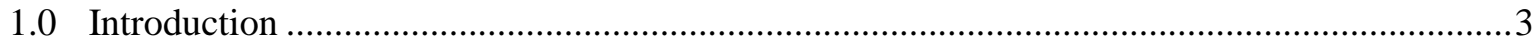

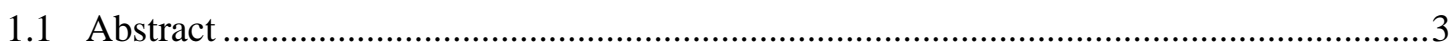

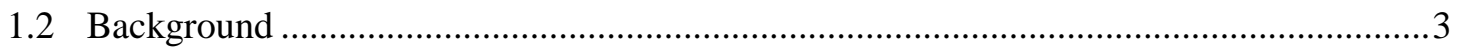

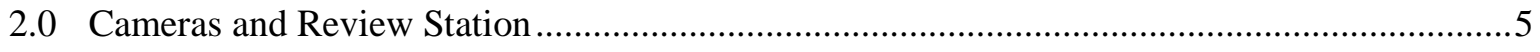

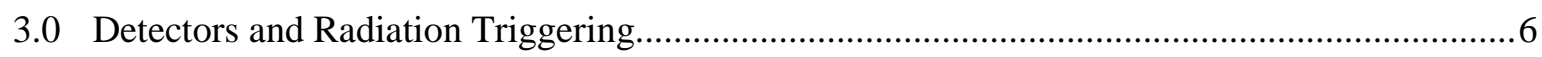

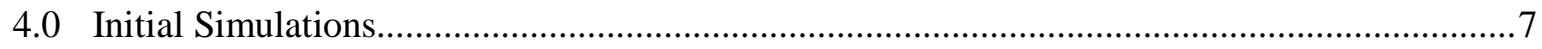

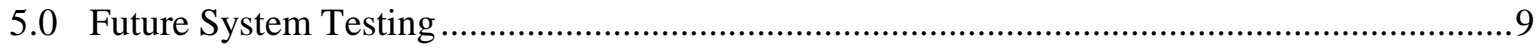

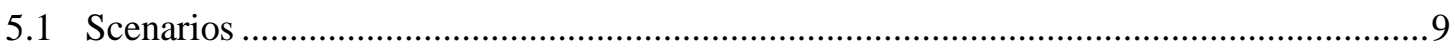

5.1.1 Radiation-Triggered Surveillance and NGRS Motion Detection............................9

5.1.2 DCM-C5-Triggered Motion Detection and Radiation-Triggered Surveillance ....10

5.1.3 Motion Detection and Radiation-Triggered Surveillance in the Same Camera ....10

5.1.4 Radiation-Triggered Surveillance followed by NGRS Motion Detection .............10

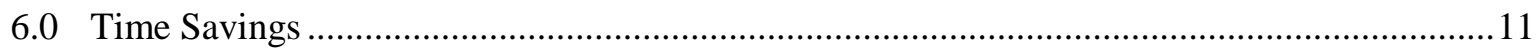

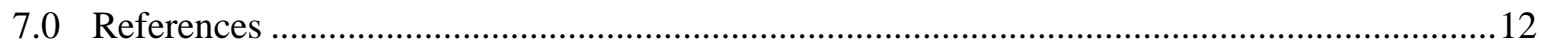

\section{Figures}

Figure 1: Surveillance Scene with 2 AOI's

Figure 2: Scenario for simulation. A 30B cylinder (blue) is moved (arrow) into the feedwithdrawal area containing several stations. The two nearest stations are occupied by additional cylinders. A $3 \times 3$ ” NaI:Tl detector (circular dashed line) is mounted near the entrance.

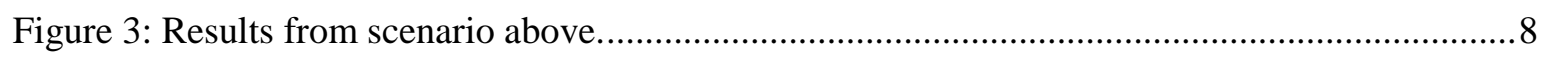

Figure 4: Notional Setup of Cameras and Radiation Detector.................................................. 


\subsection{Introduction}

\subsection{Abstract}

This report describes how the Next Generation Surveillance System cameras utilized by the International Atomic Energy Agency can be employed in areas of heavy personnel traffic provided they are triggered in a manner that distinguishes movement of uranium hexafluoride $\left(\mathrm{UF}_{6}\right)$ cylinders from movement of personnel and equipment.

\subsection{Background}

This report provides both background information and prospective strategies for testing a radiationtriggered surveillance system at an operating facility that handles $U_{6}$ cylinders. This report proffers tests designed to establish whether radiation-triggered surveillance by itself, or in combination with motion, can dramatically reduce the number of files inspectors must examine during a surveillance review.

The safeguards objectives for gas centrifuge enrichment plants (GCEPs) as outlined in the IAEA's Model Safeguards Approach for Gas Centrifuge Enrichment Plants are: ${ }^{3}$

1) Detect diversion of declared material,

2) Detect production of undeclared product from undeclared feed, and ${ }^{a}$

3) Detect production of material at enrichment levels higher than the maximum declared level reported in the Design Information Questionnaire—specifically high-enriched uranium.

To help in meeting the first two objectives, the IAEA uses short-notice inspections in combination with operator mailbox declarations. ${ }^{\mathrm{b}}$ IAEA inspectors periodically verify mailbox declarations of cylinder movements at various points throughout a site during announced and unannounced inspections and technical visits. The problem with mailbox declarations is that they consist of time-sensitive information. Inspectors verify mailboxes infrequently, because budget limitations preclude regular visits to a site for flow verification. ${ }^{\mathrm{c}}$ Visits should be random from the operators' perspective, but performing a true surprise inspection in some nations is fraught with difficulties.

Simple surveillance of $\mathrm{UF}_{6}$ cylinders cannot directly detect material diversion, but it can indicate diversion of declared cylinders or the introduction of undeclared cylinders to a process station. From a gross defect perspective, the most intractable diversion scenario involves the introduction of an undeclared feed cylinder to a process station for the production of undeclared, low-enriched uranium (LEU) product. When an operator passes an undeclared cylinder and its attendant material through a cascade, the activity can be disguised by understating the throughput of feed, product, and tails material in inventory change reports. Inspectors cannot detect undeclared throughput by verifying $100 \%$ of declared cylinders, unless they can establish the veracity of the declared total.

The IAEA recommends the use of surveillance to monitor feed and withdrawal stations. ${ }^{4}$ Surveillance cameras have the potential to provide continuous coverage by recording the passage of all cylindersdeclared and undeclared. This provides inspectors with an accurate, independent, long-term, flow balance

a. Unreported or undeclared production was formerly referred to as "excess production,” but that term is losing favor with the IAEA.

b. The term, mailbox, is shorthand for a system in which operators periodically submit cylinder flow information in a manner that cannot be edited later. The information is time-stamped and maintained in a secure database; hence, the reference to registered mail. Inspectors have the capability to review the information onsite.

c. This is also a budget burden for the national inspectors who must accompany the IAEA. 
for comparison with operator cylinder-flow declarations. The use of surveillance dramatically enhances the IAEA's ability to detect diversion of declared material and production of undeclared product from undeclared feed (objectives 1 and 2 above). ${ }^{\mathrm{d}}$

The problem with using cameras in any high-activity area involves the method of their triggering. The IAEA uses either timed intervals or motion detection to trigger its cameras. Cameras formerly used timed intervals when film was the preferred medium for surveillance. Inspectors reviewed the imagery by running the spools at high speed through a review station. Beginning in the mid-1990s, Neumann-based digital systems, using motion detection for triggering, began replacing film-and-tape-based systems. Motion sensing permits a rapid review of the entire surveillance period by eliminating successive identical frames.

Motion sensing may eliminate stagnant images, but personnel movement in continuously occupied areas can generate a plethora of motion-generated files over the course of an inspection period. A full video examination may require that an IAEA inspector and their State or Regional Authority counterpart extend the inspection by a day or more to finish the review.

Triggering cameras using changes in radiation, either in place of, or in addition to the above mechanisms, has the potential to resolve this problem. One option is to use one-or-more external radiation detectors to monitor gross gamma and/or neutron count rates at a particular point in the cylinder transport path. Cylinder movement in the vicinity of the detector, unless the cylinders are both empty and recently refurbished/new, should result in a change in the transient count rate. A sufficient change in count rate can be used to generate a trigger signal for the camera(s). Note that optimal trigger thresholds may change over time, as quiescent radiation levels fluctuate based on the number of cylinders in the vicinity.

This project presumes one early application of radiation-triggered cameras will be fuel-cycle facilities where $\mathrm{UF}_{6}$ cylinders and personnel move within the same, heavily trafficked paths. This could be the product side of a conversion facility, the feed side of a fuel fabrication plant, or the process area of an enrichment plant. An enrichment plant process area includes stations where cylinders are filled, emptied, homogenized, and blended. The process area also includes the cascade hall, but operators rarely allow cameras in the cascade hall, and even where permitted, personnel motion is minimal.

d. There is always a possibility an operator can withdraw material from a location within the cascade hall or another unmonitored removal point. Presently, only Limited Frequency Unannounced Access walkthroughs can detect such diversion. 


\subsection{Cameras and Review Station}

The Digital Camera Module (DCM-C5) is an image-generating device designed by Neumann Elektronic, $\mathrm{GmbH}$. It is the current camera module designated for the IAEA's NGSS. The IAEA is replacing virtually all its camera units with NGSS-based units powered by mains, batteries, or large networked arrays. The cameras record the numerous images generated from each motion event as a modified MPEG file. ${ }^{\mathrm{e}}$

The DCM-C5 camera has several digital inputs for external triggers along with an internal trigger for motion detection. Additional triggering sources include network commands, seal closures/openings, and an interval timer. Options include enabling or disabling any individual trigger source. Enabling multiple trigger sources permits the camera to generate a trigger event from any of them; however, the camera does not support the condition whereby multiple sources are required to trigger simultaneously.

Each trigger event generates a modified MPEG video stream. The stream consists of the image from the trigger event together with up to 8 pre-event images and 0-255 post-event images at a time-interval coincident with the current system heartbeat ( $\geq 1$ image/second). ${ }^{\mathrm{f}}$

The Next Generation Review System (NGRS) is the software that performs image decompression, authentication, and review of imagery from DCM-C5 cameras. System control and layout are similar to the General Advanced Review Software inspectors are familiar with from earlier surveillance systems, like Aquila's MOS and MUX systems. During NGRS reviews, the left-hand-side of the screen displays imagery from one or more cameras, while the right side enumerates summary information.

Neumann digital surveillance systems typically use motion detection for triggering. The camera records a motion event if successive scenes indicate there has been a sufficient change in the average luminance of tiles on the metal-oxide semiconductor (CMOS) image sensor. ${ }^{g}$ The change threshold is user-adjustable, and it typically takes several reviews to find a balance between unrecorded movements and false alarms.

The camera/NGRS combination supports two varieties of motion detection:

Front End: The user configures the camera to trigger on motion anywhere within the field of view of the DCM-C5 lens. The camera records all motion-detection events, together with preand-post-event imagery.

Back End: The user configures the DCM-C5 camera to trigger at fixed time intervals. The NGRS uses this imagery stream to generate the motion detection events. In this case triggering does not take place throughout the field of view but only within pre-defined Areas of Interest (AOIs).

It is the user's prerogative to perform motion detection within the camera or the review station. The review station provides significantly more flexibility.

Using front-end motion detection, no imagery is stored on the internal SD card or transmitted unless motion exceeds a threshold change in luminance over a weighted average of CMOS tiles. This option results in lower bandwidth transmission and smaller local storage requirements in spaces with little expected activity, such as reactor containment spent-fuel pools during reactor operation.

Back-end motion detection is the usual choice for monitoring areas where there may be periods of significant activity. To implement back-end-motion detection, the camera is set to trigger at fixed intervals. Inspectors use NGRS to perform a scene-change review of imagery based on pre-assigned

e The DCM-14, the predecessor module to the DCM-C5, recorded JPEG images. Although the compression algorithm is similar, JPEG is exclusively used for single-image compression, while MPEG can compress a video stream. As there is a plethora of redundant data in adjacent frames, MPEG need not encode the entire frame but only the differences between frames.

f. Pre-triggered images are limited to 8 at high resolution, because they are derived from a ring buffer.

g. In high-resolution mode ( $1280 \times 960)$ there are 4800 tiles on the CMOS. 
AOIs. Only regions of the screen where safeguards-relevant scene changes may occur should have AOIs assigned to them. The areas should, as much as possible, exclude the movement of personnel and nonsafeguards-related equipment. Depending on camera placement, which is not always an operator prerogative, it may not be possible to position the AOIs to exclude personnel (Figure 1). Only motion triggered events and their pre-and-post event imagery will be displayed in the NGRS; static periods will be excluded. Each AOI can be assigned its own sensitivity. A too sensitive setting can cause slight changes in shadows or reflections to trigger a scene change.

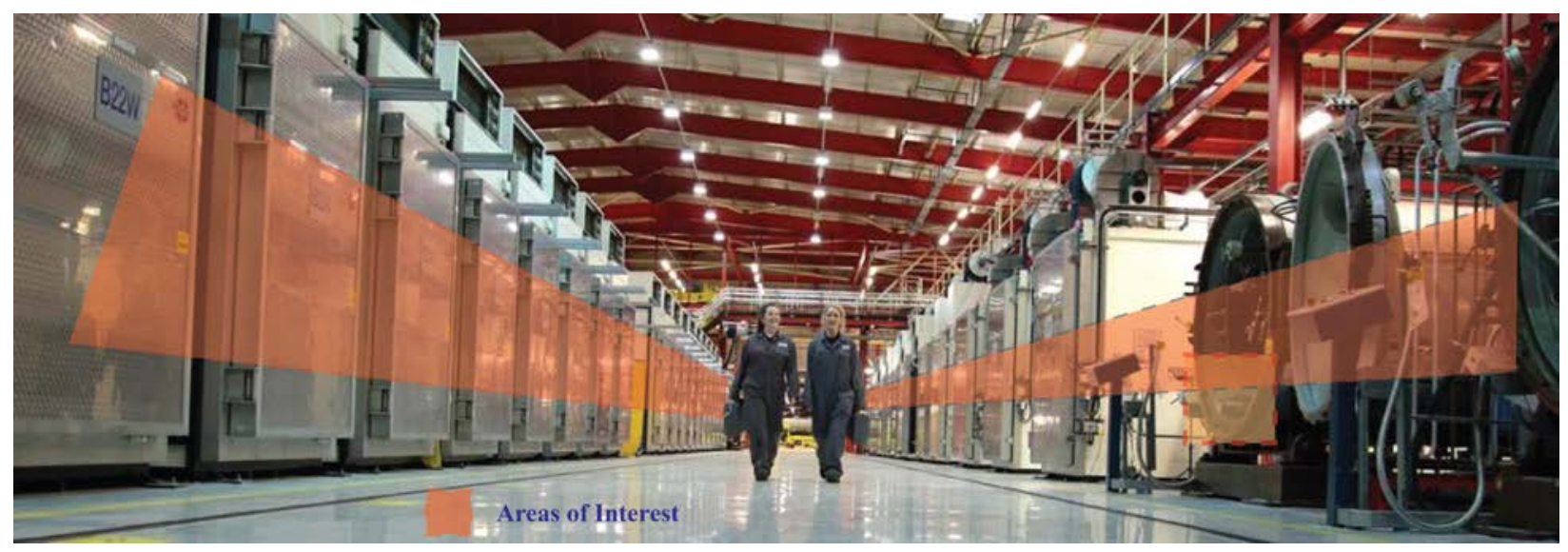

Figure 1: Surveillance Scene with 2 AOI's

Properly applied radiation triggered surveillance should eliminate the principal shortcoming of motiontriggered surveillance, which is the generation of a plethora of files generated by non-cylinder movement.

\subsection{Detectors and Radiation Triggering}

A radiation triggered surveillance system requires an external electronics package to detect radiation levels, determine significant changes in count rate, and generate a camera trigger signal. A radiation triggering component may be prototyped easily using one or more commercial radiation detectors and a programmable logic controller (PLC) to implement the necessary control logic. Verification and authentication of radiation spectra or trigger signals are separate issues the IAEA will probably consign to future tasks.

Potential radiation detectors include the following:

- Geiger-Müller (GM) tubes for gamma-ray detection and potential neutron detection

- ${ }^{3}$ He proportional counters for neutron detection

- inorganic scintillators such as $\mathrm{NaI}: \mathrm{Tl}$ for gamma-ray detection

- elpasolite scintillators such as $\mathrm{Cs}_{2} \mathrm{LiYCl}_{6}$ :Ce (CLYC) for dual gamma-ray and neutron detection

GM tubes are conventionally used for gamma-ray detection. However, the tube can be surrounded with a material that converts neutrons to gamma rays, such as dysprosium or a plastic with high hydrogen content. This would allow detection of changes in the combined gamma ray and neutron counts. Collimation or partial shielding of detectors can provide decreased sensitivity to cylinder movements outside the field of view. 
Detector output connects to a PLC that reads count rate data from the detector and implements an algorithm for generating a trigger. Ideally, the algorithm will detect all cylinder movements crossing its path, regardless of how long it takes or whether other cylinders are in the vicinity. An example algorithm would work as follows:

1) Determine the average count rates.

2) Set upper and lower trigger thresholds at the three sigma level above and below the average.

3) Wait until the instantaneous count rate falls outside the thresholds.

4) Set the trigger output high.

5) Wait for the count rates to stabilize.

6) Set the trigger output low and return to step 1.

This algorithm would not update the trigger thresholds until a trigger occurs, so that a cylinder slowly approaching the detector will still generate a trigger. Thus, trigger thresholds adapt to the presence of other cylinders in the vicinity. Trigger output remains active while count rates are changing, so the camera will capture the entire cylinder movement activity.

\subsection{Initial Simulations}

We performed a rough order-of-magnitude simulation to quantify the expected count rate from a representative scenario. In this scenario (Figure 2), a $3 \times 3$ ” NaI:Tl detector is installed on the floor before the entrance of a cascade hall. The detector is shielded on three sides to remove background counts. A full 30B, 5\% enriched, $\mathrm{UF}_{6}$ cylinder is moved past the detector at walking speed. The cascade hall contains multiple stations, but we assume only the first two are occupied with additional cylinders. We estimate other stations will be shielded by these first two. Although the model has low fidelity, it provides some idea of the expected count rate.

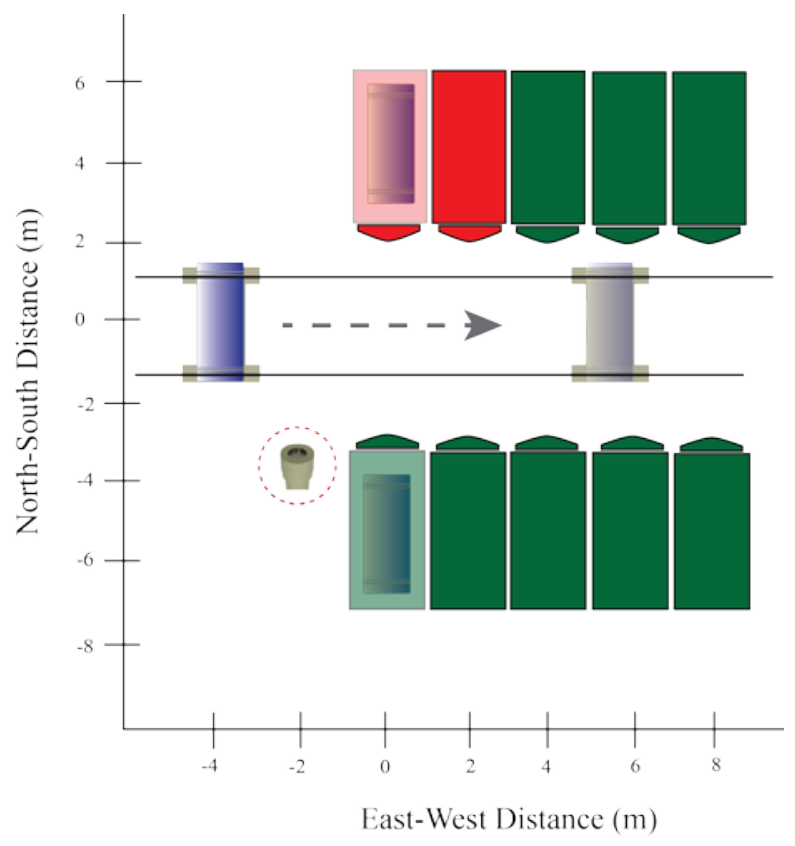

Figure 2: Scenario for simulation. A 30B cylinder (blue) is moved (arrow) into the feed-withdrawal area containing several stations. The two nearest stations are occupied by additional cylinders. A $3 \times 3$ ” NaI:Tl detector (circular dashed line) is mounted near the entrance. 
We then used GADRAS ${ }^{\mathrm{h}}$ to simulate the total gamma count rates for this scenario. ${ }^{5}$ Based on prior experience, the results may be off by a factor of two compared to measured data, but the accuracy is sufficient for a preliminary analysis. We used a sample time of one second and aligned the point of closest approach with the boundary between two samples to represent the worst-case condition. The resulting time profile contains a well-defined peak (Figure 3).

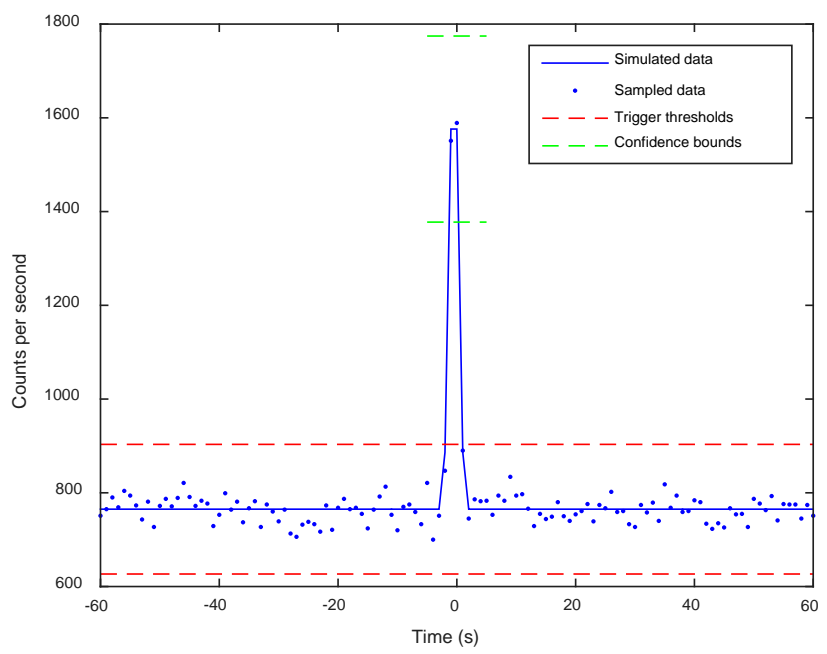

Figure 3: Results from scenario above.

Indicated on the plot are the trigger thresholds at five standard deviations from the quiescent count rate (red), along with the confidence bounds at five standard deviations from the peak count rate (green). Because the lower green line is far from the upper red line, cylinder movement would be detected in essentially all cases.

One could also consider mounting the detector on the ceiling, above the entrance to the cascade hall. This configuration removes the detector from the floor, but introduces concerns with regard to the weight of the shielding. We found that the sensitivity in this case is somewhat lower due to the increased distance between the detector and the cylinder. Removing the shielding degrades the sensitivity further.

We also simulated a $1 \times 1$ " detector but found count rates were too low to detect cylinder movement. A GM tube would likely have even lower sensitivity. Thus, we recommend the $3 \times 3$ ” NaI:Tl detector as a baseline option for the radiation-triggered surveillance system. The extra sensitivity is desirable since most cylinders will have lower levels of enrichment.

We plan to continue the evaluations in future work after defining more specific detection requirements for the radiation-triggered surveillance system.

h. The Gamma Detector Response and Analysis Software (GADRAS) applies a detector response function to compute the output of gamma-ray and neutron detectors when exposed to radiation sources. The response function is fundamental to the ability to compute the response of a detector to a known source. 


\subsection{Future System Testing}

The ideal facility where a prototype radiation triggered surveillance system should be tested is one in which there are:

- one or more process areas in which there are frequent movements of $U_{6}$ cylinders, personnel, and various equipment items. Candidate locations include the feed and withdrawal area of a GCEP, the output of a conversion facility, and the conversion side of a fuel fabrication facility

- cylinders of various enrichments with assorted quantities of $U_{6}$ including some having only small quantities of material adhering to the walls of an otherwise empty cylinder

Testing will necessitate the use of at least two DCM-C5 cameras. Two cameras are requisite to permit viewing of the same events by both cameras using different triggering methods. Camera placement should allow them to have the same field of view (Figure 4). Three cameras would be ideal, with the third camera providing a wide-angle view of the entire cylinder handling area, which may stretch 30 meters or more. The third camera would by internally triggered using short intervals to record all area activity over the entire testing period. Image transfer to the review station requires a local network or SD card removal.

For all scenarios, it would be beneficial to perform additional tests with stationary $\mathrm{UF}_{6}$ cylinders in assorted locations to alter the background radiation level.

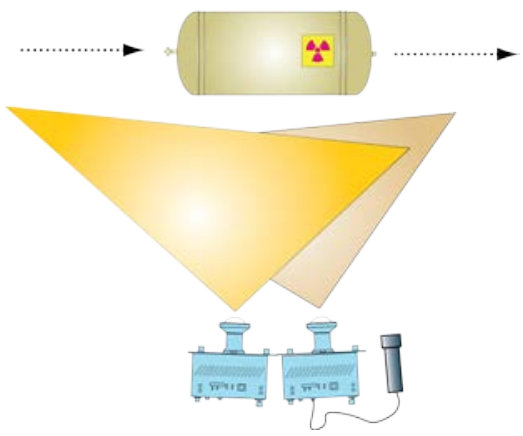

Figure 4: Notional Setup of Cameras and Radiation Detector

\subsection{Scenarios}

Below are some prospective scenarios. The assortment is not exhaustive, because the flexibility of the DCM-C5/NGRS combination can accommodate many others.

\subsubsection{Radiation-Triggered Surveillance and NGRS Motion Detection}

$$
\begin{aligned}
& \text { Camera }{ }^{\#} 1 \text { records images at timed intervals. Inspectors transfer the } \\
& \text { imagery to NGRS for scene-change detection. } \\
& \text { Camera }{ }^{\#} 2 \text { records images triggered by changes in radiation }
\end{aligned}
$$

In this scenario, camera ${ }^{\#} 1$ will record a series of field-of-view images at the camera's heartbeat interval. At an interval of 10-seconds, this will total over 250,000 images in the course of a month. The NGRS scene-change algorithm will retain only the motion-change images within the assigned AOIs. Depending on area activity and the size and location of AOIs, NGRS may retain thousands of scene-change files for review.

Camera 2 will record cylinder flow based on changes in radiation flux. Cylinder flow recorded by camera ${ }^{\#} 2$ will then be compared with cylinder flow recorded by camera ${ }^{\#} 1$. In an area with significant personnel activity, the imagery recorded by Camera ${ }^{\#} 2$ should be significantly less than that recorded by 
camera ${ }^{\#} 1$ due to the exclusion of non-cylinder-related movement. Discrepancies related to missing cylinder movements can be resolved by viewing the raw, time-sequenced imagery from Camera ${ }^{\#} 1$. $^{\mathrm{i}}$

The imagery from camera ${ }^{\#} 1$ can be re-run by the NGRS algorithm using different AOIs and a modified dynamic threshold for scene changes. Any changes made to the radiation triggering algorithm for camera ${ }_{2} 2$ will require an additional field test.

\title{
5.1.2 DCM-C5-Triggered Motion Detection and Radiation-Triggered Surveillance
}

\author{
Camera ${ }^{*} 1$ records imagery triggered by scene changes within the \\ camera \\ Camera ${ }^{\#} 2$ records imagery triggered by changes in radiation
}

In this scenario, motion will trigger camera ${ }^{\#} 1$, whenever there is activity within its entire field of view. As no AOIs can be set within the camera, it will record motion events based on a user-defined dynamic threshold. In this instance, it would be best for Camera ${ }^{\#} 1$ to have a narrow field of view. Camera ${ }^{\#} 2$ will be triggered by an external input from a radiation sensor. Neither camera will record timed-interval surveillance of the area over the entire time period.

This scenario is probably not the best option for full-time operation, as the NGRS is capable of performing a more flexible scene-change review. Nevertheless, it can estimate the disparity in image generation between cylinder movements and all movements over the period in question. Depending on the facility, the difference may be two orders of magnitude or more. Any changes to the scene-change sensitivity or the radiation trigger algorithm will require an entirely new field test to demonstrate the modification.

\subsubsection{Motion Detection and Radiation-Triggered Surveillance in the Same Camera}

\author{
Camera ${ }^{*} 1$ records imagery triggered by changes in radiation \\ Camera ${ }^{\#} 2$ records imagery from simultaneous radiation triggers and \\ scene changes
}

This scenario will require that an external motion sensor be coupled to the radiation triggering circuit for camera ${ }^{\#} 2$ in a Boolean “AND” configuration, because the motion trigger signal of the DCM-C5 is not accessible externally and cannot be combined with the radiation trigger signal on board the camera. The advantage of employing both motion detection and radiation triggering in the same camera is that it should generate the least number of images for inspector review. In the event no working system utilizes this option, the ${ }^{\#} 1$ vs. ${ }^{\#} 2$ camera comparison is important for establishing the efficacy of stand-alone radiation triggering.

\subsubsection{Radiation-Triggered Surveillance followed by NGRS Motion Detection}

\section{Camera ${ }^{\#} 1$ records imagery triggered by changes in radiation \\ Camera ${ }^{\#} 2$ records imagery at timed intervals}

This scenario performs an NGRS scene-change review of the imagery garnered from camera ${ }^{\#} 1$, which triggers on radiation changes only. The difference between imagery garnered from camera ${ }^{\#} 1$ and the imagery culled from the scene change review should be little to none. This is an optional test intended to verify the efficiency of a single camera set to trigger on radiation. The output from camera ${ }^{\#} 2$ is intended only as check of total cylinder throughput.

i Embedded in the video stream are Meta-data: including camera, date and time, and trigger source as MPEG user data. Data extraction yields an XML document that includes an imagery table of contents. 


\subsection{Time Savings}

In a moderately active area, NGSS cameras triggered by motion detection are capable of storing months of high-resolution cylinder movement information; consequently, inspectors do not have to travel frequently to a site to verify mailbox information but can wait until a scheduled inspection to review material flow activities. Mailboxes are a good tool for verifying cylinder passage over short intervals, but as the intervals increase, it becomes increasingly difficult to verify them. Frequent mailbox verifications are at odds with IAEA budget limitations. Cameras in the process area provide a means of verifying throughput that does not require frequent visits or a continual onsite inspector presence.

Enrichment plants require greater travel costs relative to other facilities because of frequent equipment modifications and repairs, last-minute cylinder verifications prior to shipment, Limited Frequency Unannounced Inspections, and longer interim and physical inventory verifications. Surveillance usage can minimize the use of Limited Frequency Unannounced Inspections because verification of recent flow activity is not required; although, it would not negate cascade walkthroughs and swipe samplings.

Depending on camera placement, AOI locations, and process-area arrangement, motion-triggered video should record all activities associated with connecting and disconnecting cylinders to the process; whereas, radiation triggering should capture only cylinder movements. This should considerably reduce inspector review time (perhaps as much as two orders of magnitude), because connecting and disconnecting cylinders to the process is labor-intensive activity. For example, to empty a cylinder: ${ }^{6}$

- The cylinder is moved on a cart to the feed station.

- The valve is rotated to the 12-o'clock position

- The cylinder is placed in the feed station and a pigtail is used to connect the cylinder valve to the piping manifold

- Autoclave door is closed

- The connection is pressure tested and evacuated

- The cylinder valve is opened. The cylinder is heated, and cylinder pressure is monitored. Once sufficient pressure has built up, it is valved into the system. When pressure can no longer be maintained the valve is shut.

- The autoclave door remains shut for days while the cylinder cools

- The cylinder is disconnected and removed from the process area 


\subsection{References}

1. IAEA Board of Governors. Implementation of the NPT Safeguards Agreement and relevant provisions of Security Council resolutions in the Islamic Republic of Iran, GOV/2015/65 para. 22, November 18, 2015.

2. INMM. Cooley, J. "Model Safeguards Approach and Innovative Techniques Implemented by the IAEA at Gas Centrifuge Enrichment Plants." Proceedings of the Institute of Nuclear Materials Management INMM 48th Annual Meeting Tucson, AZ, July 8-12, 2007.

3. INMM. Cooley, J. "Model Safeguards Approach and Innovative Techniques Implemented by the IAEA at Gas Centrifuge Enrichment Plants.” Proceedings of the Institute of Nuclear Materials Management INMM 48th Annual Meeting Tucson, AZ, July 8-12, 2007.

4. INMM. Cooley, J. "Model Safeguards Approach and Innovative Techniques Implemented by the IAEA at Gas Centrifuge Enrichment Plants.” Proceedings of the Institute of Nuclear Materials Management INMM 48th Annual Meeting Tucson, AZ, July 8-12, 2007.

5. Mitchell, D., Harding, L., Thoreson, G., Horne, S.; GADRAS Detector Response Function, Sandia National Laboratory, SAND2014-19465, November 2014.

6. USEC. Uranium Hexafluoride: A Manual of Good Handling Practices. United States Enrichment Corporation, USEC-651, January 1995. 



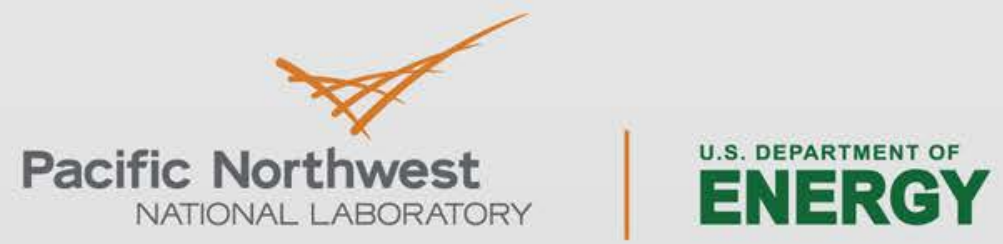

Proudly Operated by Battelle Since 1965

902 Battelle Boulevard

P.O. Box 999

Richland, WA 99352

1-888-375-PNNL (7665)

www.pnnl.gov 